\title{
Extending an Educational Math Game with a Pedagogical Conversational Agent: Facing Design Challenges
}

\author{
Björn Sjödén ${ }^{1}$, Annika Silvervarg ${ }^{2}$, Magnus Haake $^{3}$, and Agneta Gulz ${ }^{1}$ \\ ${ }^{1}$ Lund University Cognitive Science \\ \{Bjorn.Sjoden, Agneta.Gulz\}@lucs.1u.se \\ ${ }^{2}$ Department of Computer and Information Science, Linköping University \\ Annika.Silvervarg@liu.se \\ ${ }^{3}$ Department of Design Sciences, Lund University \\ magnus.haake@design.1th.se
}

\begin{abstract}
We describe our work-in-progress of developing an educational game in mathematics for 12-14 year olds, by adding social and conversational abilities to an existing "teachable agent" (TA) in the game. The purpose of this extension is to affect cognitive, emotional and social constructs known to promote learning, such as self-efficacy and engagement, as well as enhancing students' experiences of interacting with the agent over an extended period of time. Drawing from the EnALI framework, which states practical design guidelines, we discuss specific design challenges and exemplify research considerations as to developing the agent's visual representation and conversational module. We present some initial findings from prototype testing with students from the target group. Promising developments seem to reside in pronouncing the agent's personality traits and expanding its knowledge database, particularly its range of conversational topics. Finally we propose some future studies and research directions.
\end{abstract}

Keywords: pedagogical agents, conversational agents, teachable agents, social learning, educational game, EnALI.

\section{Introduction}

Learning "in real life" is, arguably, always connected to some form of social interaction or communicative purpose with other human beings. Many educational researchers have noted that learning is an essentially social activity, and also that we are more likely to succeed in our efforts to educate if we recognize this principle [e.g. 1, 2, 18, 43]. But can we capture the social qualities of learning when students sit to learn on their own, in front of a computer?

In our on-going work of developing an educational game in mathematics for children, we have sought to address precisely that question. By including a pedagogical conversational agent in the game, we set out to create opportunities for social interaction between students and agents within the learning environment. We are presently 
working on enriching the social dimension of the game by developing the agent's conversational capabilities as well as its visual embodiment.

The first and foremost motivation behind this extension is to make individual students who use the digital learning environment benefit from social interactions known to promote learning in natural environments, by increasing motivation, building selfefficacy and acknowledging new skills as they are building up. As to the effect of motivation on (reflective) cognition, Schank and Neaman [37] list three issues: the participation issue, as to whether students participate at all in a learning activity, the indexing issue, as to the way memories are organized, and the attention issue, as to the quality of attention to what should be remembered.

The pedagogical agent, then, should keep students engaged in the learning environment, without attracting too much attention to itself as an artifact (vis-à-vis the learning material). By elaborating the agent's social features, although it is clearly non-human, we capitalize on fundamental human tendencies for what makes a task engaging and cognitively worthwhile. For example, Chen and colleagues presented neurophysiological evidence that the very belief of a social experience activates the brain's reward circuitry, which helps cement newly learned associations [12, 13].

At the same time, one would like to utilize the possibilities of a designed digital environment to minimize detrimental social influences (such as distractors and negative feedback) that may also be at work in the real world. This goes hand-in-hand with an overall approach of presenting a subject domain (e.g. mathematics) in a generally more appealing and defusing context in order to attract a wider group of students. As will be exemplified below, the question of aesthetics must not go overlooked when designing a virtual learning environment: It has been demonstrated that visual appeal can influence both perceived usability [38] and user satisfaction, in terms of the perceived quality of interaction [29]. In the words of interaction designer and theorist Donald Norman, "attractive things work better" [32].

A second motivation for the extension is that we are looking to expand the target user group, from 7-12-year-olds, for whom a basic version of the game has been available, to 12-14 year-olds. Considering the greater computer and gaming experience of older students, they are likely to have higher expectations on both visual aesthetics and the complexity of interaction in the learning environment. With maturity comes also the ability to handle more complex interactions and (at least potentially) greater responsibility in their social role.

In particular, we focus on the development and social role of a Teachable Agent (TA) $[8,11]$, which is a form of AI-based educational technology that builds upon the pedagogy of "learning by teaching" $[1,3]$. There are a number of reasons why students would learn more efficiently with a TA than without a TA in an otherwise identical game setup. A core factor is the so-called "protégé effect" [11]. That is, students tend to take more responsibility and put more effort into teaching someone else than into learning for themselves, while at the same time being able to ascribe failures and errors to the agent, rather than to personal shortcomings. In the following we describe how we design the TA as a social agent and how we conceive of the present challenges in working towards specific design decisions. We exemplify methods used and present some initial evaluations with target users. 


\section{Background}

Previous research shows that pedagogical agents can be beneficial for learning, provided an appropriate - pedagogical and visual - design [5, 20, 23]. It is therefore imperative that one carefully considers the particular pedagogical and social role of a particular agent in a given environment, for example whether it be a mentor, a coach or a peer. Whereas visual appearance has not been a traditional focus of research on pedagogical agents, recent reviews have deemed agents' visual features important value for affecting motivation and engagement in the learning environment where it is employed [4, 22, 25]. Not the least, an appropriately designed agent may serve as a 'virtual role model', depending on how its represented social features (e.g. attractiveness, style, gender, age, etc) correspond to students' explicit and implicit preferences (e.g. stereotypes) $[5,23,28,35]$. This idea is in line with Bandura's classical work on social modeling $[1,2]$ as well as research on stereotypes within social cognition, which suggests that one needs only perceive few, salient characteristics of a person to activate complex mental representations about him or her [10]. When having the opportunity of a prolonged interaction, the content and quality of conversation may add to or detract from this impression.

One major beneficial function of a purposefully designed pedagogical agent is then that students may assimilate a range of positive behaviors and attitudes in line with an attractive social model represented by the agent, for instance a "cool and successful peer in school" in the context of learning mathematics. Exploiting visual characteristics and conversation to communicate positive social stereotypes offer means of cueing model behavior in a way that is directly and effortlessly understood by the student. However, it is difficult to make straight-off comparisons between different learning environments due to the wide variety of contexts in which pedagogical agents are used [39]. We will therefore describe our learning environment in more detail, and elaborate on the visual and verbal aspects of what makes the "peer"-like social qualities of our $\mathrm{TA}$ in the game.

\subsection{The Learning Environment: A Learning-by-Teaching Mathematics Game}

Our work extends an educational math game named "The Squares Family", developed by Lena Pareto and Daniel Schwartz $[33,34]$. The game specifically trains basic arithmetic skills, such as carry-overs and borrowings, with focus on grounding base10 concepts in spatial representations. To do so, the game employs a board-game design with "playing cards" and a common game board, with a variety of game modes and levels of difficulty. All arithmetic operations are made visually explicit using graphical metaphors of colored squares and boxes that can be "packed" or "unpacked", in numbers of 10 . The aim is to pick the card from a given set (visible to both players) that in combination with what is represented on the game board maximizes the number of carry-overs (in the addition games) or borrowings (in the subtraction games). See example screenshot in Fig. 1. 


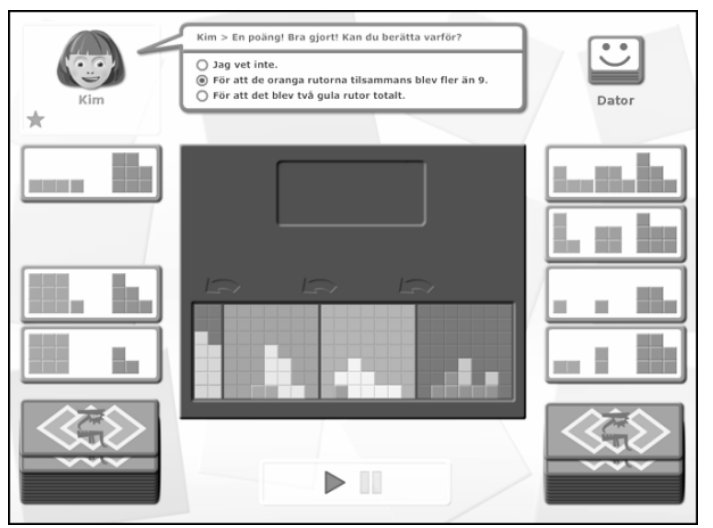

Fig. 1. Screenshot of an early version of the extended math game (actually in color). Here, the TA, called Kim, is set in a "Watch and learn"-mode against the computer (Swedish: "Dator"). Kim asks why the player received a point (star) for picking a particular card, the answer being "Because the orange squares together became more than 9".

The game includes a TA which can be put in different modes. In "Watch and learn"-mode, the TA picks up on game rules as the student responds to multiplechoice questions on particular tasks and game moves during game-play. A typical question from the TA would be "Why did you pick this card?". The student chooses between listed potential explanations (but only one correct answer), including a "don't know" option. Proper (or improper) choices of cards and answers promote corresponding skills in the TA throughout the game. In "Try and play"-mode, the agent can be set to suggest game cards, which the student can confirm by clicking "Ok" or deny by suggesting another card. Finally, in "Play Self"-mode, the agent is set to play a session of the game automatically (against the computer or another agent or human player), allowing the student to watch its performance.

In a recent study, we found empirical support for learning benefits of using a TA in this game: Compared to a control class of children the same age in the same school, which did not play the game, children who did play the game over an extended period of time improved both in performance on standardized math tests and in confidence in explaining math to a peer [24]. These findings have encouraged us to look further into ways of strengthening the student-agent bond, so as to be socially engaging, motivating and fun, in relation to the learning material.

\subsection{Expanding on the Pedagogical Agent as a Social Being}

Not much is needed for a human being to respond socially to an obviously non-human system. As evidenced by the much quoted and vastly demonstrated "media equation", formulated by Reeves and Nass [36], humans tend to treat computers as real social actors - for example, by responding rudely to a malfunctioning ("rude") computer, or praising a program which presents a positive evaluation of the user. This holds true even in the complete absence of a human-like representation (e.g. a photo or picture of a person, let alone a virtual agent). It is therefore not surprising that also the 
rudimentary agent (TA) of the original math game (depicted to the left in Fig. 2) elicits social responses, something we observed in our previous studies of students playing the math game in school [30].

Compared to human-human interaction, the original TA still leaves vast room for enriched social cueing, through elaborated visual features, animations, movement and verbal expressions. We maintain that expanding on the social dimension will affect not only the direct interaction with the agent, but how students approach the game as a whole, even affecting their decision (when free to decide) whether to play the game at all. The "media equation" is not limited to people's responses to a particular interaction with a computer, but tends to generalize to how they approach similar contexts and informational content [36]. We reason that students gaining a positive experience from engaging in the math game are more likely to play it again, and providing social interaction with the agent offers means to pick up on and respond to students' attitudes which otherwise remain unexpressed.

Elaborating the agent's social abilities both constrains and allows greater control of students' social responses: not only do we want to promote certain effects (such as affecting the student's mood) but also control the direction of effects (making the student happy). We will return to the challenges of actually meeting these criteria in subsequent paragraphs; first we want to present the tools we use for the purpose. In short, we conceive of elaborating the agent's social profile in a two-fold modular development, which consists of (1) Visual representation of the pedagogical agent, and (2) Conversational abilities of the pedagogical agent.

Visual representation of the pedagogical agent. Elaborating the visual design of the agent essentially targets two aspects of students' approach to the interaction: one is to provide visual cues for the constraints as to what students might expect from interacting with the agent (e.g. "will this be a cool or nerdy character to talk to?"); another is simply to increase likeability by pleasant looks and graphics, including a visual makeover of its original game environment.

A guiding question for our design considerations has been: "Judging by looks, whom would the students like to interact with?" An important choice in this regard was that of a cartoon-like character over naturalistic or semi-naturalistic looks. First, cartoon-like agents have been argued to make students accept limitations more readily [17]; second, they lend themselves better for animation of simple affective cues, such as smiles and head tilts. Fig. 2 depicts the development from the first rudiment of an agent via two iterations, involving students of the relevant age group.
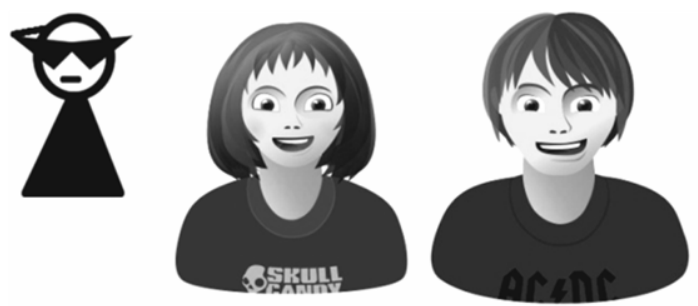

Fig. 2. Visual representation of the pedagogical agent, revised following student input. The original, rudimentary agent image (left) was replaced by one female and one male alternative. 
Conversational abilities of the pedagogical agent. The second main extension under development is a module for allowing free (not multiple-choice-steered) written conversation between the student and the TA. We make a primary distinction between on-task dialogue, which is constrained by the present math tasks and takes a multiplechoice format (see 2.1 for examples), and off-task dialogue, which takes the form of a chat window, open to freer conversation. The off-task dialogue content, in turn, can be distinguished into on-domain conversation related to school, math, the math game, etc., and off-domain conversation, related to any other topics. These distinctions are important for our design considerations, when it comes to the pedagogical functions of the social chat in relation to the learning content we want to communicate.

So, one might ask, what are the pedagogical benefits of dedicating a conversational module for topics not relating to mathematics in a math game? Again, the answer resides in what makes learning engaging and effective in real life. Our present developments of the math game strive to keep certain influence within rather than outside the educational game environment. Considering the agent's role as a type of colearner and peer, it seems vital to promote the student's sense of factors such as trust in and rapport with the agent.

Bickmore [6], among others, has shown that including anecdotes and narrative storytelling can contribute to building rapport with a conversational agent, besides having a positive effect on people's general interest in interacting with it. Possibly connected to this, is the effect of "small talk" (irrespective of content) as offering some cognitive rest from arduous educational tasks. Finally, broad conversational abilities of the TA - particularly referring to the transitions between off-domain and on-domain topics - are hypothesized to help overcome another stumbling-block of math education in school, namely to make the subject of mathematics more attractive by reframing math tasks in a defusing and appealing context. This makes part of the strategy to have conversational content improve students' attitudes and self-efficacy for math. Besides enhancing the subjective learning experience, it has been demonstrated for a variety of learning material that such motivational constructs can have a vital impact on objective performance [2, 18, 31, 45].

\section{Guiding Framework: Enhancing Agent-Learner Interaction (EnALI)}

Turning to the questions of implementation, one might ask how one reaches a specific design decision among the myriad of possible alternatives. The research literature indicates a particular need for formulating guidelines as to how an effective communication can be achieved between a student and an agent, in order to cause as little frustration and conflict as possible. What should then our social, pedagogical and conversational agent look and behave like?

Whereas there is no theoretical framework to answer this question in detail, there are sufficient research-based and empirical findings not to leave all new design decisions to "designer's intuition" or other personal bias. The most comprehensive, recent and practical set of guidelines we have found to meet our present design needs, is the Enhancing Agent-Learner Interaction (EnALI) framework [41]. 
In short, the EnALI guidelines are divided into three design foci - user interaction, message, and agent characteristics - that the authors ground on socio-cultural notions of learning [43], cooperative learning [27], and conflict theory [15]. User interaction refers to the ways the agent and the student cooperate and relate to each other; message refers to the verbal information the agent sends to the student, and agent characteristics focuses on those variables that inherently define the agent. For the complete set of references to the empirical findings on which particular guidelines are based, we refer to the original EnALI article by Veletsianos, Miller and Doering [41]. An overview of the actual design guidelines is provided in Table 1.

Table 1. The Enhancing Agent-Learner Interaction framework, from Doering, Miller and Veletsianos [34]

\begin{tabular}{|c|c|c|}
\hline & Design focus & Guidelines \\
\hline 1 & $\begin{array}{l}\text { User } \\
\text { interaction }\end{array}$ & $\begin{array}{l}\text { Agents should be attentive and sensitive to the learner's } \\
\text { needs and wants by: } \\
\text { - Being responsive and reactive to requests for additional } \\
\text { and/or expanded information. } \\
\text { - Being redundant. } \\
\text { - Asking for formative and summative feedback. } \\
\text { - Maintaining an appropriate balance between on- and off- } \\
\text { task communications. }\end{array}$ \\
\hline 2 & Message & $\begin{array}{l}\text { Agents should consider intricacies of the message by: } \\
\text { - Making the message appropriate to the receiver's } \\
\text { abilities, experiences, and frame of reference. } \\
\text { - Using congruent verbal and nonverbal messages. } \\
\text { - Clearly owning the message. } \\
\text { - Making messages complete and specific. } \\
\text { - Using descriptive, non-evaluative comments. } \\
\text { - Describing feelings by name, action, or figure of } \\
\text { speech. }\end{array}$ \\
\hline 3 & $\begin{array}{l}\text { Agent } \\
\text { characteristics }\end{array}$ & $\begin{array}{l}\text { Agents should display socially appropriate demeanor, } \\
\text { posture, and representation by: } \\
\text { - Establishing credibility and trustworthiness. } \\
\text { - Establishing role and relationship to user/task. } \\
\text { - Being polite and positive (e.g., encouraging, } \\
\text { motivating). } \\
\text { - Being expressive (e.g. exhibiting verbal cues in } \\
\text { speech). } \\
\text { - Using a visual representation appropriate to content. }\end{array}$ \\
\hline
\end{tabular}

The challenges we face by adding the particular agent in the math game demonstrate how the EnALI guidelines sometimes overlap and influence one another when applied in practice. This is especially apparent in our overall challenge of designing a complex social character. For example, "Agent characteristics" can hardly be completely separated from the "Message" which the agent conveys in social (off-task) 
conversation, as regards expressing a certain personality. As to "User interaction", one must keep in mind that our pedagogical agent (especially considering the nature of a teachable agent; see 4.1 below) does not primarily serve for information retrieval and is not positioned in an "expert" or "guiding" role.

In the following section, we highlight three main design challenges that correspond to different aspects in the EnALI framework, before addressing the influence from actual students by empirical testing and observations. It should be made clear that these challenges and the approaches we take in meeting them, like the EnALI guidelines, are presented under separate headlines of expository reasons but must be viewed within the inter-related whole of the math game as a learning environment.

\section{Implementation Challenges}

\subsection{The Agent's Knowledge Profile: What Should the Agent Know?}

One of the first things students will ask themselves when being presented with the opportunity to chat with a pedagogical agent is what they can talk to the agent about. This poses a wide and conspicuous challenge of construing a knowledge database that can meet most student requests while keeping within the necessary limits of developers' time and resources. As such, this challenge applies to practically all conversational agents and similar systems, but there are certain aspects that both constrain and expand this challenge for the particular agent we aim to develop.

On the one hand, although students generally need to experience a pedagogical agent as being useful for information purposes for the learning task at hand [39], not all agents are equally important for obtaining specific information. In our case, because we employ a teachable agent, it is ostensibly "non-knowledgeable" in its subject domain (mathematics) from the beginning and can thus respond to information requests in this domain by referring to the fact that "you are supposed to teach me". This assumes, of course, that the agent's role as "teachable" is properly introduced to the student, and that there is a functional, underlying AI architecture that implicitly keeps track of what the agent learns throughout the game and reflects this in ensuing conversations. In other words, the agent is allowed to be perceived as unknowledgeable, but not as unteachable.

On the other hand, the challenge of achieving a sufficiently comprehensive knowledge database becomes considerably more complex when making the agent more of a social actor, that is, when adding an off-task conversational module. According to the EnALI guidelines, messages should be "complete and specific" while being "appropriate to the receiver's abilities, experiences, and frame of reference". For an agent that supposedly should be able to converse about any topic, both the specificity and completeness of messages must be limited. In addition, there is an apparent risk of clashes as to the knowledge the agent exhibits in one area vis-à-vis another, for example, if it demonstrates good knowledge about school, but no knowledge about mathematics. Besides, the application does not contain any AI module for learning other domains than the math game. We conceive of this challenge as a need for carefully defining specific knowledge areas corresponding to students' interests, while balancing the complete knowledge profile of the agent over the on-task-off-task spectrum. 


\subsection{The Agent's Social Profile: How Should the Agent Communicate?}

While the challenge of defining a knowledge profile corresponds to "what" the agent should know, the agent's social profile corresponds to "who" it should be and "how" it should communicate its knowledge. The EnALI guideline that the agent should be "polite and positive", in terms of being encouraging and motivating, refers to the demonstrated "politeness effect" of conversational agents [44]. However, an allthrough polite and nice agent may be perceived as predictable and boring, especially if students are to interact with it repeatedly and over an extended period of time [cf. 40]. After all, we are used from everyday social interactions that people vary in exhibited moods and states of mind, but also that they have a consistent personality. A conflict might thus arise between predictability and consistency as regards the social behavior of the agent. Again, there are obvious limits to the complexity which can be construed for a digital character. At the same time, this challenge should be viewed in relation to students' expectations and experience of other non-human agents (e.g. characters in animated movies, computer games and other virtual agents), rather than to the full spectrum of a human personality.

In possible contrast to the expected politeness of the agent stands the potential (anti-)social behavior of the student. Previous studies have addressed the risk that, when students are assigned to freely converse with an agent, they sometimes engage in verbal abuse of the agent $[9,42]$. In one (possibly extreme) case with a female conversational agent in a social science application, evaluations showed that only $5 \%$ of student comments were task-related and as much as $40 \%$ of comments were abusive (sexually explicit) [42]. Although a wide range of conversational subjects is wished for, there are clearly certain subjects one wishes to avoid from a social as well as from a pedagogical point of view. The risk of verbal abuse may be considered a sub-challenge to the social profile which is subject to the social affordances of the agent and the interaction situation.

\subsection{Off-Task Engagement: Too Much or Too Little?}

We propose one particular EnALI guideline as a separate challenge, concerning user interaction: "Maintaining an appropriate balance between on- and off-task communications". Due to the complexity of both knowledge and social features of our pedagogical agent, both the appeal and non-appeal of engaging in conversation with it may be problematic. It should be added, that in our game, students are not completely free to choose when to interact with the agent. A related challenge is then how to regulate the opportunities for agent-student off-task interaction from a system level.

First, the balance between on-task and off-task activities can be disturbed if offtask conversation becomes more engaging than the learning activities themselves. This has been noted to be the case for other agents allowing both on-task and off-task conversation, even to the point that students become completely immersed in the offtask conversation [40].

Second, there are studies which show that some students simply are not interested in off-task conversation, but prefer to treat the agent as exclusively a resource in working towards solving a task [21]. Then the potential pedagogical benefits of offtask conversation previously proposed will take no effect. Besides, under-engagement 
in off-task conversation makes it more difficult to capture students' attitudes and qualitative remarks during the game, including which tasks they find difficult, what they enjoy, when they become tired, etc.

\section{Present Approaches and Initial Findings}

We consider facing the challenges identified above - and reducing them to a manageable complexity - as largely a question of managing students' expectations on the agent's abilities. By that we mean to frame and guide the interaction with the student in such a way that, ideally, the shortcomings and knowledge gaps of the agent never become a critical issue for achieving an effective communication. As mentioned, one way of constraining expectations by visual means in the initial stage, was to opt for a cartoon-like character which carry less associations to the full capabilities of a "real" human being.

Similarly, carefully designed dialogue strategies may be used to frame expectations on what the agent is able to converse about, for example by using expressions and syntax corresponding to that of the target students' social group. Having the agent represent a member of a certain age group (say an 11-year-old for the interaction with 12-14-year-olds) is one approach to constraining the range of students' initiatives to likely conversational topics by both visual and conversational means [cf. 5]. In the case of a TA, it also makes sense to represent the agent as slightly younger and less advanced than the actual students, yet intelligent, in order to bring forward its "teachable" affordances [8, 11].

It seems clear that the EnALI guidelines we work from require further specification when it comes to actual implementation. Also "students' expectations" need to be clarified and concretized for different aspects of the interaction: What could students expect from an 11-year-old, virtual teachable peer? Can it share interesting views and comments? Does it appear outgoing, funny, natural, strange, cocky? To get a grip on these and related questions, we initiated an iterative design process involving representatives from the target user group in the following five steps:

1. The researchers compiled a preliminary sketch of the agent's personal profile, including a visual representation and an intentionally unspecific description of the agent's personality and interests (see 2.2 and Fig. 2).

2. The sketch was evaluated using focus groups of 4-5 target students $(\mathrm{N}=20)$, who were asked to review the profile, give feedback and suggest conversational topics with the agent. Importantly, the students expressed what they desired that a conversational agent of this type be like, in terms of exhibited "personality" traits.

3. Following student input, a testing procedure was conducted with a revised agent profile, such that students $(\mathrm{N}=7)$ worked in pairs on different computers, where one student acted as the agent in simulated off-task conversation. The dialogue logs were collected and used to identify conversational topics (e.g. "music", "school", "friends") as indicative of what an actual student-agent conversation would comprise.

4. Prototype 1 of the agent was developed, using AIML, focusing on off-domain topics and a simple mixed-initiative dialogue strategy. Students $(\mathrm{N}=38)$ tested the prototype in the context of playing the game by chatting with the agent on topics of their own 
choice. As a follow-up, we used a questionnaire based upon the SASSI (Subjective Assessment of Speech System Interfaces) [26]. The questionnaire listed 25 statements relating to the subjective experience of using the system, for example "I rather play the game than talk to the agent" and "It felt natural to talk to the agent", rated on a 7-point Likert scale. The "personality" of the agent prototype was formally measured using a Swedish translation and adaptation of the TIPI (Ten Item Personality Inventory) test, which is based on the Big Five (or Five-Factor Model) personality dimensions [19].

5. Prototype 2 of the agent was developed on basis of corpus analysis of the dialogue logs and additional focus groups, expanding the agent's range of conversational topics and dialogue history. The prototype was tested on students $(\mathrm{N}=43)$ using the same procedure as in testing of the first prototype, using the same questionnaires.

\subsection{Preliminary Conclusions Relating to the Agent's Knowledge Profile, Social Profile and Off-Task Engagement}

From analysis of the SASSI questionnaires the most indicative item for students enjoying the conversation was "I had many things to talk to the agent about". This item strongly correlated to "I liked talking to the agent" $(r=0,84, p<0,01)$ and "It was fun talking to the agent" ( $r=0,77, p<0,01)$. Still, the results indicated large individual differences that call for further investigation. The results from the TIPI test, which show the change in scores from prototype 1 to prototype 2 compared to the desired personality ratings proposed by the focus groups, are summarized in Table 2.

Table 2. Student mean ratings of how they desired and perceived the personality traits of the agent, including the difference between prototypes 1 and 2, as assessed on a 7-point Likert scale $(1=$ not at all pronounced, $7=$ very pronounced $)$

\begin{tabular}{lcccc}
\hline Personality trait & Desired & Proto. 1 & Proto. 2 & Diff \\
Extraversion & 6,00 & 4,33 & 4,99 & $+1,01$ \\
Agreeableness & 6,04 & 4,39 & 4,98 & $+1,06$ \\
Emotional Stability & 5,54 & 4,91 & 4,95 & $+0,59$ \\
Openness to Experience & 5,29 & 4,16 & 4,42 & $+0,87$ \\
Conscientiousness & 5,25 & 3,94 & 4,05 & $+1,20$ \\
\hline
\end{tabular}

Some clues as to what makes an engaging conversation with the agent were given by relating the agent's preferred knowledge areas (interests) to its desired personality. Expanding the agent's range of conversational topics seems a fruitful direction to take for achieving positive student experiences and engagement. In this respect, the developments point in the right direction, as the agent's personality is becoming more expressive and clear to the students. The initial findings from the iterative designimplementation-evaluation process referred above also point to some preliminary conclusions for meeting the implementation challenges of designing the agent's knowledge profile and social profile, as well as affecting students' engagement in offtask conversation.

The agent's knowledge profile. Some of the personal interests and possible off-task conversational topics listed by the researchers in the preliminary sketch (step 1 above) were confirmed, such as wanting to talk about friends and school in general. But there 
were also some presumed topics that students did not bring up, such as film and traveling. The focus groups consistently added topics of interest to the list: sports, music, and computer games. Following prototype 1 and analysis of this new corpus, revealed a number of additional common topics: food, animals and particular school subjects. Existing topics were also refined with subtopics, for example "music" with artists and songs and "school" with teachers. The following iteration (prototype 2) resulted in only one additional topic: television. A more detailed, qualitative analysis of the collected dialogue corpus remains to be done, including an analysis of possible gender differences as to preferred topics of conversation.

The agent's social profile. The focus groups confirmed that the agent should be friendly, curious, eager to learn, and like school, which aligns well with previous findings. However, it was added that the agent should not be too polite, but express some 'attitude'. This is one example of how a particular user group can differ from general design guidelines, and again emphasizes the importance of involving target students in the design. The specific design decision of age and gender in relation to the target group, we assume, would have particular effect on students' tendencies to abuse the agent (e.g. a cartoon-like agent representing an 11-year-old boy for male students would seem a less likely target of abuse than the attractive, adult female agent used in a previous study addressing this issue [42]). Naturally, there are more explicit means of setting students' expectations on the interaction, such as having the agent express that it is not interested in certain topics or has no knowledge about them.

Off-task engagement. A proper analysis of engagement in on-task versus off-task conversation (and the subdivision into off-domain and on-domain topics) will need to await further development of the conversational module. As to regulating the time spent on off-task interactions, we plan to have the agent take the initiative to conversation at particular intervals during game-play, and state that "now, the break is over, we need to return to the game" if too much time is devoted to social chatting (e.g., more than 5 minutes at a time). Besides, the student may deny an offer to chat with the agent. To our knowledge, such a mixed-initiative approach to student-agent interactions is unusual, and its effects on engagement in a game setting have not been previously evaluated in the literature.

\section{Overall Conclusions and Future Work}

One important lesson from the EnALI framework as a whole is that equal attention should be kept to the learner and the agent, implying that it would be fruitless to work on the agent out of context or lacking knowledge about the intended students (e.g. 1214-year-olds) and the way they communicate. This makes a complexity of challenges that must also be taken into account for anyone in search for "straight" solutions to resolving agent-student conflicts of the types we discuss here. That is why we actively involve students from the target group at an early stage of the design process, and do not draw any definite conclusions by relying solely upon the framework as to the design decisions we make. At present, there appears a notable gap between what the students think the agent should be like and what they actually perceive it is like. This 
has led us to plan at least two more iterations and prototypes, targeting more advanced dialogue strategies and manipulation of verbally expressed personality traits.

Our approach of iteratively and continuously refining the design, until an effective and engaging learning environment is reached, corresponds to the explicit objectives stated by the EnALI for extending the framework. Student input, through questionnaires and observations, continuously serves as a basis for design decisions taken by the researchers and developers. Actual learning effects of selected implementations will be assessed using a research design with matched school classes playing the math game during math class (i.e. "experimental groups") compared to classes not playing the game, but following the regular educational curriculum (i.e. "control groups"). Theoretically, we aim at refining the ways in which specific, goal-relevant cognitive processes are triggered by social interventions.

We close this paper by proposing three planned studies into the effects on learning, attitudes and engagement in relation to the concerns we have raised. These are:

1. The value of off-task conversation. Evaluating effects of using the off-task conversational module in the game, compared to game-play without it.

2. Differential effects of gender representations. Evaluating differential effects for both sexes of students when using more or less pronounced masculine and feminine (including androgynous) agent looks. This will address the agent-student relation in terms of power (as reflected in dialogue) and the student's role as a teacher (as reflected in the agent's competence level).

3. Effects of types of conversational content. Exploring the effects from manipulating linguistic styles (e.g. the length of utterances, lexical and syntactical choices) and introducing, versus not introducing, content such as anecdotes and small talk relating to mathematics.

Acknowledgments. This research project was supported by the Knowledge Foundation (KK-stiftelsen). The authors would like to thank the two anonymous reviewers for constructive comments on a previous version of this paper.

\section{References}

1. Bandura, A.: Social foundations of thought and action: a social cognitive theory. PrenticeHall, Englewood Cliffs (1986)

2. Bandura, A.: Self-efficacy: The foundation of agency. Lawrence Erlbaum Associates Publishers, Mahwah (2000)

3. Bargh, J.A., Schul, Y.: On the cognitive benefits of teaching. Journal of Educational Psychology 72, 593-604 (1980)

4. Baylor, A.L.: Promoting motivation with virtual agents and avatars: role of visual presence and appearance. Philosophical Transactions of the Royal Society B 364, 3559-3565 (2009)

5. Baylor, A., Rosenberg-Kima, R., Plant, E.: Interface agents as social models: The impact of appearance on females' attitude toward engineering. In: CHI 2006 Extended Abstracts on Human Factors in Computing Systems, pp. 526-531. ACM, New York (2006)

6. Bickmore, T.: Relational Agents: Effecting Change through Human-Computer Relationships. Ph.D Thesis, Media Arts \& Sciences, Massachusetts Institute of Technology (2003) 
7. Biswas, G., Katzlberger, T., Brandford, J., Schwartz, D.: TAG-V.: Extending intelligent learning environments with teachable agents to enhance learning. In: Moore, J.D., Redfield, C.L., Johnson, W.L. (eds.) Artificial Intelligence in Education, pp. 389-397. IOS Press, Amsterdam (2001)

8. Blair, K., Schwartz, D.L., Biswas, G., Leelawong, K.: Pedagogical agents for learning by teaching: Teachable Agents. Educational Technology Special Issue on Pedagogical Agents 47, 56-61 (2007)

9. Branham, S., De Angeli, A.: Special issue on the abuse and misuse of social agents. Interacting with Computers 20, 287-291 (2008)

10. Brewer, M.: A dual process model of impression formation. In: Wyer, R., Srull, T. (eds.) Advances in Social Cognition 1, pp. 1-36. Erlbaum, Hillsdale (1988)

11. Chase, C., Chin, D., Oppezzo, M., Schwartz, D.: Teachable Agents and the Protégé Effect: Increasing the Effort Towards Learning. J. Sci. Educ. Technol. 18, 334-352 (2009)

12. Chen, J., Shohamy, D., Ross, V., Reeves, B., Wagner, A.D.: The impact of social belief on the neurophysiology of learning and memory. In: Annual Meeting of the Society for Neuroscience, San Francisco, CA (2009)

13. Davachi, L., Mitchell, J.P., Wagner, A.D.: Multiple routes to memory: Distinct medial temporal lobe processes build item and source memories. Proceedings of the National Academy of Science 100(4), 2157-2162 (2003)

14. De Angeli, A., Brahnam: I hate you! Disinhibition with virtual partners. Interacting with Computers 20, 302-310 (2008)

15. Deutsch, M.: The resolution of conflict: Constructive and destructive processes. Yale University Press, New Haven (1973)

16. Doering, A., Veletsianos, G., Yerasimou, T.: Conversational Agents and their Longitudinal Affordances on Communication and Interaction. Journal of Interactive Learning Research 19, 251-270 (2008)

17. Dowling, C.: Intelligent agents: some ethical issues and dilemmas. In: Proceedings of AIC 2000, pp. 28-32. ACS, Canberra (2000)

18. Dweck, C.S.: Self-theories: Their Role in Motivation, Personality and Development. Psychology Press, Philadelphia (1999)

19. Gosling, S.D., Rentfrow, P.D., Swann Jr., W.B.: A very brief measure of the big five personality domains. Journal of Research in Personality 37, 504-528 (2003)

20. Gulz, A.: Benefits of virtual characters in computer based learning environments: claims and evidence. International Journal of Artificial Intelligence in Education 14, 313-334 (2004)

21. Gulz, A.: Social enrichment by virtual characters - differential benefits. Journal of Computer Assisted Learning 21, 405-418 (2005)

22. Gulz, A., Haake, M.: Design of animated pedagogical agents - a look at their look. International Journal of Human-Computer Studies 64, 322-339 (2006)

23. Gulz, A., Haake, M.: Challenging gender stereotypes using virtual pedagogical characters. In: Goodman, S., Booth, S., Kirkup, G. (eds.) Gender Issues in Learning and Working with Information Technology: Social Constructs and Cultural Contexts, pp. 113-132. IGI Global, Hershey (2010)

24. Gulz, A., Lindström, P., Haake, M., Pareto, L., Sjödén, B.: A Teachable Agent Based Game Affording Collaboration and Competition - Evaluating Math Comprehension and Motivation (2010) (submitted)

25. Haake, M., Gulz, A.: A look at the roles of look \& roles in embodied pedagogical agents a user preference perspective. International Journal of Artificial Intelligence in Education 19, 39-71 (2009) 
26. Hone, K.S., Graham, R.: Towards a tool for the subjective assessment of speech system interfaces (sassi). Natural Language Engineering 6, 287-305 (2000)

27. Johnson, D., Johnson, R., Holubec, E.: Cooperation in the Classroom, 6th edn. Interaction Book Company, Edina (1993)

28. Kim, Y., Wei, Q., Xu, B., Ko, Y., Ilieva, V.: MathGirls: Increasing Girls' Positive Attitudes and Self-Efficacy through Pedagogical Agents. Paper presented at 13th International Conference on Artificial Intelligence in Education. AIED, Los Angeles (2007)

29. Lindegaard, G., Dudek, C.: What is this evasive beast we call user satisfaction? Interacting with Computers 15, 429-452 (2003)

30. Lindström, P., Gulz, A., Haake, M., Sjödén, B.: Matching and mismatching between the pedagogical design principles of a math game and the actual practices of play. Journal of Computer Assisted Learning (in press)

31. Maddux, J.: Self-Efficacy: The Power of Believing You Can. In: Snyder, C.R., Lopez, S.J. (eds.) Handbook of Positive Psychology, pp. 277-287. Oxford University Press, New York (2005)

32. Norman, D.: Emotion \& design: attractive things work better. Interactions 9, 36-42 (2002)

33. Pareto, L.: The Squares Family: A Game and Story based Microworld for Understanding Arithmetic Concepts designed to attract girls. In: World Conference on Educational Multimedia, Hypermedia and Telecommunications 1, pp. 1567-1574 (2004)

34. Pareto, L., Schwartz, D., Svensson, L.: Learning by guiding a teachable agent to play an educational game. In: Proceeding of the International Conference on Artificial Intelligence in Education, pp. 662-664. IOS Press, Amsterdam (2009)

35. Plant, E.A., Baylor, A.L., Doerr, C., Rosenberg-Kima, R.: Changing middle-school students' attitudes and performance regarding engineering with computer-based social models. Computers \& Education 53, 209-215 (2009)

36. Reeves, C., Nass, B.: The Media Equation. Cambridge University Press, New York (1996)

37. Schank, R., Neaman, A.: Motivation and failure in educational simulation design. In: Forbus, K., Feltovich, P. (eds.) Smart Machines in Education, pp. 37-69. AAAI/MITPress, Menlo Park, CA (2001)

38. Tractinsky, N., Katz, A., Ikar, D.: What is beautiful is usable. Interacting with Computers 13, 127-145 (2000)

39. Veletsianos, G.: Contextually relevant pedagogical agents: Visual appearance, stereotypes, and first impressions and their impact on learning. Computers \& Education (in press)

40. Veletsianos, G., Miller, C.: Conversing with Pedagogical Agents: A Phenomenological Exploration of Interacting with Digital Entities. British Journal of Educational Technology 39, 969-986 (2008)

41. Veletsianos, G., Miller, C., Doering, A.: EnALI: A Research and Design Framework for Virtual Characters and Pedagogical Agents. Journal of Educational Computing Research 41, 171-194 (2009)

42. Veletsianos, G., Scharber, C., Doering, A.: When Sex, Drugs, and Violence Enter the Classroom: Conversations between Adolescent Social Studies Students and a Female Pedagogical Agent. Interacting with Computers 20, 292-302 (2008)

43. Vygotsky, L.S.: Mind in Society: The development of higher psychological processes. Harvard University Press, Cambridge (1978)

44. Wang, N., Johnson, W.L., Mayer, R.E., Rizzo, P., Shaw, E., Collins, H.: The politeness effect: Pedagogical agents and learning outcomes. International Journal of Human Computer Studies 66, 96-112 (2008)

45. Zimmerman, B.J., Schunk, D.H.: Self-regulated Learning and Academic Achievement. Lawrence Erlbaum Associates Publishers, Mahwah (2001) 